\title{
Propagation of Seizurelike Activity in a Model of Neocortex
}

\author{
Running title: Seizurelike Activity in a Model of Neocortex
}

\author{
Wim van Drongelen, ${ }^{1,2}$ Hyong C Lee, ${ }^{1}$ Rick L. Stevens, ${ }^{2,3}$ \\ and Mark Hereld ${ }^{2,3}$
}

\footnotetext{
${ }^{1}$ Department of Pediatrics, The University of Chicago, Chicago, IL

${ }^{2}$ Computation Institute, The University of Chicago, Chicago, IL

${ }^{3}$ Mathematics and Computer Science Division, Argonne National Laboratory, Argonne, IL
}

\section{Contact Information:}

Wim van Drongelen

Department of Pediatrics, C372, MC 3055

The University of Chicago

5841 S. Maryland Avenue, Chicago, IL. 60637-1470.

Tel: (773) 8349049

Fax: (773) 7024786

E-mail: wvandron@peds.bsd.uchicago.edu

Presented during the international conference "An Overview of Epilepsy Research: What, Where, When and Why," organized by the Center for Integrative Neuroscience and Neuroengineering Research (CINNR), May 19-20, 2006, in Chicago. 


\begin{abstract}
Seizures in pediatric epilepsy are often associated with spreading, repetitive bursting activity in neocortex. We examined onset and propagation of seizurelike activity using a computational model of cortical circuitry. The model includes two pyramidal cell types and four types of inhibitory interneurons; each neuron is represented by a multicompartmental model with biophysically realistic ion channels.

We determined the role of bursting neurons and found that their capability of driving network oscillations is most prominent in networks with either weak or relatively strong excitatory synaptic coupling. Synaptic coupling strength was varied in a separate set of simulations to examine its role in network bursting. Oscillations both between cortical layers (vertical oscillations) and between cortical areas (horizontal oscillations) emerge at moderate excitatory coupling strengths. For horizontal propagation, existence of a fast conducting fiber system and its properties are critical.

We conclude that seizurelike oscillatory activity may originate from single neurons or small networks and that activity may propagate in two principal fashions: one that can be represented by a unidirectional (pacemaker)-type process and the other as multi- or bidirectional propagating waves. The frequency of the bursting patterns relates to underlying propagating activity that can either sustain or disrupt the ongoing oscillation.
\end{abstract}

\title{
Keywords:
}

Neocortex, Epilepsy, Ictal Activity, Seizure Propagation, Neocortical Microcircuitry, Computational Model. 


\section{Introduction}

Epilepsy is a neurological disorder characterized by chronic seizures. It affects $\sim 50$ million people worldwide, and in a large ratio $(>30 \%)$ of these patients, the disease is seriously affecting the quality of life because their seizures cannot be adequately controlled with medication (e.g., Kwan and Brodie 2000). A major factor explaining the limited success of therapy in epilepsy is the lack of understanding of the processes leading to seizure onset. Recordings of brain electrical activity reveal that most seizure types are associated with rhythmic activity; and because these ictal patterns can usually be distinguished in the electroencephalogram (EEG), the activity must involve several square centimeters of the cerebral cortex (Tao et al. 2005). Simultaneous extra- and intracranial recordings of the onset of partial seizures demonstrate initiation of seizure activity in one or few intracranial electrodes, followed by propagation of the ictal pattern to a larger area that is commonly (but not always) associated with the onset of clinical signs. The prevailing opinion among basic and clinical neurophysiologists is that hypersynchrony and hyperactivity of neuronal networks underlie epileptiform activity (e.g., Delgado-Escueta et al. 1999). Although these assumptions seem reasonable and may, in fact, be correct in many seizure types, the general validity of these hypotheses has recently been questioned on the basis of results obtained in studies of both animal epilepsy models and computational models (e.g., Netoff and Schiff 2002; van Drongelen et al. 2003, 2005; Barela et al. 2006).

The advantage of a seizure model approach, especially of computational models, is that properties can be varied and measured, thus allowing insight in interrelationships between individual functions that govern the process. During the past few decades, Traub and co-workers (e.g., Traub and Llinas 1979; Traub and Miles 1991; Traub et al. 1994) developed and validated a model of a hippocampal pyramidal cell that was based on multiple, coupled cylindrical compartments in which realistic ion channels were included. Willis et al. (1993) and Klopp et al. 
(1998) described large-scale network models of the hippocampus. In these studies, the compartmental cell models described by Traub's group were simplified by reduction of the number of compartments for computational reasons. Most published models involving neocortex focus either on intrinsic properties of the neuron (Desthexe and Pare 1999; Fleidervish et al., 1996; Kepecs et al. 2002; Wang 1999) or on small and simplified networks (Golomb 1998; Golomb and Amitai 1997; Lytton and Sejnowski 1991; Van Drongelen and Hecox, 2003). Lytton et al (1997) modeled epileptiform oscillations in thalamocortical networks. Recently, however, researchers have attempted to model cortical columns or larger neocortical networks, including relatively high levels of cellular detail enabled by a parallel computing approach (van Drongelen et al. 2004, 2005; Traub et al. 2005; Migliore et al. in press).

The purpose of this study is to examine the propagation of seizure activity in neocortical circuitry. We use an existing computational model that is capable of generating seizurelike discharges (Van Drongelen et al. 2005). The neural network in this model includes the excitatory and inhibitory cell populations with a multicompartmental representation of each cell type. The network connectivity includes the essence of neocortical microcircuitry: recurrent excitation, negative feedback loops, feedforward loops, disinhibition, and direct electrical contact between interneurons (Amitai et al. 2002). We show that seizurelike activity may be sustained and propagate between neuronal populations, located either in different cortical layers within a restricted area or in different cortical areas. We find that the propagation of local seizurelike activity to significant portions of the cortex can take place in a pacemaker fashion, where a cell or small network imposes pathological behavior on a larger population, or in a bidirectional fashion, where cells or networks are connected reciprocally, generating a sustained oscillation between the players involved in the process.

\section{Methods}


A model including excitatory and inhibitory neuronal populations and connectivity based on neocortical histology (e.g., DeFelipe et al. 2002; Mountcastle 1997; Nieuwenhuys 1994), was used to study propagation of seizure-like activity. Details of the model were published previously (Lee et al. 2005; van Drongelen et al. 2004, 2005); the following is a short summary of the essential features. The excitatory network (Fig. 1A) consists of superficial pyramidal cells from layers $2 / 3$ (5 compartments) and deep pyramidal cells from layers $5 / 6$ (7 compartments). The inhibitory cells (2 compartments) receive input from both types of pyramidal neurons. Gap junctions between inhibitory cells show nearest neighbor connectivity (e.g., Amitai et al. 2002). The implemented inhibitory interneurons are three types of basket cells and a chandelier cell. The basket cell types inhibit the pyramidal cell soma, whereas the chandelier cell inhibits directly on the initial segment (Fig. 1B). In a short range, the synaptic connectivity decreases with distance between source and target elements (e.g., Hellwig 2000; Feldmeyer and Sakman 2000; Krimer and Goldman-Rakic 2001; Nieuwenhuys 1994). At the intermediate and long range ( $>1 \mathrm{~mm}$ and up to a centimeter scale), fast connections create a so-called small-world network. Synaptic connections were simulated with a dual exponential function using the values described in Traub and Miles (1991). Extracellular activity was initially obtained as a weighted sum of currents generated by the model neurons in a preliminary simulation study (Nunez 1981). At present the model can generate spontaneous and evoked activity at the compartmental, cellular and network scales. These activity patterns include seizurelike bursting activity (van Drongelen et al. 2005).

The computational model is implemented with the parallel GENESIS neural simulator (Bower and Beeman 1998) to facilitate a high degree of detail, large-scale simulations, and multiple parameter searches. A scalable version of the model runs on the Jazz computing cluster at Argonne National Laboratory, which enabled us to perform $>10$ years of single-processor simulation within a two-year period.

\section{Results}




\section{Vertical Oscillation}

An example of collective behavior of a model containing 656 neurons during a reduction of the excitatory coupling strength is shown in Fig. 2A. During a reduction of the excitation, an irregular bursting pattern at higher levels of synaptic coupling transforms in a rapid oscillation, followed by sets of large-amplitude bursts at low levels of transmission strength. The effect of removing bursting neurons on the network trace is shown in Fig. 2B. At higher and lower levels of excitatory coupling, the presence or absence of the bursting neurons affects the network behavior, whereas the rapid oscillations at moderate levels occur in both panels $\mathrm{A}$ and $\mathrm{B}$. The effects of selectively reducing the excitatory connectivity either between pyramidal cells or between pyramidal neurons and inhibitory cells are shown in Fig. 2C, D. Although some oscillation remains in Fig. 2C (reduced coupling strength between the pyramidal cell populations), in both cases the network pattern changes significantly relative to the trace in Fig. $2 \mathrm{~A}$.

Details of the neural activity underlying the oscillatory behavior are shown in Fig. 3. The trace in Fig. 3A represents the weighted sum of all neuronal activity, representing the EEG picked up by a macroelectrode at the surface of the cortex. The traces in Fig. 3B show the averaged activities of different neuronal populations. During the repetitive bursting phase of the network activity, the superficial (S_PYR) and deep pyramidal (D_PYR) cell populations oscillate at the same frequency as the EEG, but there is a phase difference of $\sim 180^{\circ}$ between them. In other words, from the viewpoint of these populations, the activity is propagated back and forth. Further, we found that each burst in the pyramidal cell population is associated with a burst in the inhibitory neurons; this is shown for the type 1 basket cell population (B1) in Fig. 3C. The vertically oriented oscillation between the pyramidal cell layers occurs at a frequency of $\sim 28 \mathrm{~Hz}$. Because of the nature of the process, this frequency is determined by the time required for the activity to bounce back and forth. This time interval is determined by only a few model parameters: 
(a) signal transmission between neurons ( $\sim 4 \mathrm{~ms})$,

(b) distance $(1.1 \mathrm{~mm})$, and

(c) speed of propagation (average: $80 \mathrm{~mm} / \mathrm{s}$ ) between the pyramidal cell layers.

The time interval for propagation back and forth is approximately $(2 \times 1.1) / 80=27.5 \mathrm{~ms}-$ the added time for transmission in both layers added results in a time interval of $\sim 27.5+2 \times 4 \approx 35.5$ ms - corresponding to an oscillation frequency of $\sim 28 \mathrm{~Hz}$. Consequently we were able to lower the model's oscillation frequency by reducing the conduction velocity or by increasing the layer interdistance (data not shown).

The effect of reduced synaptic excitation is further dissected in a set of simulations shown in Fig.3. The excitation reduction can be subdivided into a reduced synaptic effect from the excitatory neurons on the excitatory cells, on the inhibitory cells, or on both. The result when both populations are affected is shown in Fig. 3A. Reduction of synaptic coupling strength to the excitatory and inhibitory populations separately is shown in Fig. 3B, C. In both cases the oscillations are destroyed. In the case of reduced excitatory $\rightarrow$ excitatory coupling (Fig. 3B), some oscillatory effects at a lower frequency remain. Oscillations disappear with the reduced effect on the inhibitory neurons (Fig. 3C). These results confirm our earlier finding that both excitatory and inhibitory populations participate in sustaining the network activity (Fig. 3B, C). A major question in observing network behavior is to what extent intrinsic and network factors contribute.

\section{Horizontal Oscillation}

Assuming that the model network shown in Fig. 3 can represent a focal area, we studied propagation in a horizontal direction. Lee et al. (2005) reported entraining in a model in which two patches of cortical tissue were placed at an interdistance of $3 \mathrm{~mm}$ was reported earlier. Here we show the effect of the focus on a follower patch with either reciprocal connectivity between the patches or a one sided connectivity from focus to follower (Fig. 4). Similar to the oscillation 
in the vertical direction, the conduction speed plays a critical role in the horizontal spread of activity. Without any fast connections there is no propagation and the follower patch is quiet (Fig. 4A). Propagation of the focal oscillation occurs when the bidirectional fast connections are present at speeds of $0.22 \mathrm{~m} / \mathrm{s}$ (Fig. 4B, E), $0.85 \mathrm{~m} / \mathrm{s}$ (Fig. 4D, E), and $15 \mathrm{~m} / \mathrm{s}$ (Fig. 4E). Unsurprisingly the oscillation is strong when the intrinsic horizontal oscillation's frequency is the same as the vertical oscillation of $\sim 28 \mathrm{~Hz}$ corresponding with a period of $35.5 \mathrm{~ms}$. Since the patches are separated by $3 \mathrm{~mm}$ (resulting in a path of $2 \times 3=6 \mathrm{~mm}$ for activity to bounce back and forth), and since a cellular transmission of $\sim 4 \mathrm{~ms}$ is used (the same as used above for the vertical oscillation), the expected optimal horizontal conduction velocity that supports the vertical oscillation with a period of $35.5 \mathrm{~ms}(28 \mathrm{~Hz})$ is $6 /(35.5-2 \times 4) \approx 0.22 \mathrm{~m} / \mathrm{s}$. At relatively high conduction speed, the oscillations are also sustained because the activity that is reflected from the follower back to the focus arrives too fast to have an effect because the focus is still active or refractory. In these cases making or breaking the synaptic contacts from follower to focus does not have a large effect on the follower's activity (left and right panels in Fig. 4D). At a conduction speed around $0.22 \mathrm{~m} / \mathrm{s}$, breaking the reciprocal connectivity doesn't have a large effect either (Fig. 4B). In contrast to the effects we observed at low and high conduction velocity, most of the oscillations both at the focal and follower patches are destroyed at horizontal conduction speeds around $0.34 \mathrm{~m} / \mathrm{s}$ (Fig. 4C). In this case breaking the reciprocal connection has a large effect - the oscillation is now propagated to the follower (Fig. 4C, left panel). From the details shown in Fig. 4E one can see that, if oscillations are propagated, the phase difference between focal and follower circuits decrease with speed.

\section{Discussion}

We report that a computational model, representing neocortical circuitry with compartmental neuronal representations with biophysically realistic ion channels, can generate and propagate seizurelike activity. At higher and lower levels of synaptic transmission bursting 
cell types generate network bursts that become periodic at low levels of coupling strength, while at moderate levels of transmission, the oscillations are rapid and occur independent of presence of bursting neurons (Fig. 2A, B). In a simulation of an active isolated focal patch (Fig. 3) we showed that the frequency of oscillation at moderate levels of connectivity can be determined from the neuronal transmission, the conduction velocity, and the distance between the layers. In the scenario where this patch is a focus entraining another cortical patch (Fig. 4), the intrinsic/focal oscillation must be sustained, or the process will terminate. For extending the oscillation from focus to follower, a fast fiber system is required (Fig. 4A). In the simulations shown in Fig. 4, we sustain and propagate the oscillation at speeds of $0.22 \mathrm{~m} / \mathrm{s}$ and at speeds of $0.85 \mathrm{~m} / \mathrm{s}$ or higher. If the focal and follower are connected both ways, a propagation velocity of $0.34 \mathrm{~m} / \mathrm{s}$ terminates the oscillation in both the focal and follower patches (Fig. 4C). From the simulation results, we summarize with the following conclusions.

1. Network oscillations between layers (vertical oscillation) may be generated and sustained by bursting cells, or (if the level of excitatory connectivity is moderate) they may exist without bursters (Fig. 2A, B). In any of the cases an ongoing oscillation forms the basis of the propagating activity (Fig. 5A).

2. Fast connections are essential for a focal area to entrain a follower (horizontal propagation), (Fig. 4).

a. If the fast connections propagate the activity in both directions - as is likely to occur in neocortex with multiple connections - both vertical and horizontal oscillations must coexist. A bidirectional fast connection between focus and follower may propagate seizurelike oscillations if it sustains the focal activity by matching the focal frequency (Fig. 4B) or by establishing a connection that is so fast that the reflected activity arrives during the refractory period of the focal area (Fig. 4D). In these cases the presence of absence of bidirectional connectivity slightly changes but does not make or break the entrainment process: there is a slight amplification of focal oscillation in Fig. 4B, and a 
reduction of focal activity in Fig. 4D. At even higher speeds the effect caused by the recurrent activity from follower to focus is close to zero ( $\mathrm{v}=15 \mathrm{~m} / \mathrm{s} \pm 95 \%$, Fig. 4E). In cases where the entrainment process is unidirectional or bidirectional but without an essential role for the reflected activity, we effectively may represent the propagation as a unidirectional, pacemaker-type process (Fig. 5B), in the other case as a bidirectional one (Fig. 5C).

b. A bidirectional connection will terminate the oscillation in both focus and follower at particular combinations of speed and neuronal transmission time (e.g., Fig. 4C). In this case, bi- or unidirectional connections make a critical difference. That is, if the oscillations occur at the wrong frequencies, they may annihilate each other. The bidirectional connection plays a critical role (Fig. 5C).

The reason seizure activity is relatively robust for the duration of the event is perhaps due to the fact that the process involves waves of activity propagating between populations rather than between individual single cells. At any point in time each population involved in the oscillation has multiple neurons at slightly different levels of excitation; that is, there will always be a set of neurons ready to propagate the activity (Fig. 5D). In addition, a fiber system that connects oscillating neuronal populations most likely possesses a range of conduction velocities capable of supporting a spectrum of oscillation frequencies (Fig. 5D).

An important question is how representative the seizurelike activity in the computational model is for epileptiform activity in real circuitry. Our model demonstrates that periodic bursting may occur at relatively low levels of excitatory coupling. The commonly held opinion is that seizures are caused by increased strength of the excitatory synapse, and experimental evidence supporting this view exists; for instance, Steriade and Amzica (1999) found that the amplitude of evoked EPSPs around seizure onset is increased and the latency decreased. Although our findings contradict the opinion that seizures result from hyperexcitability, there is also experimental 
evidence that certain types of seizures in neocortex and hippocampus may indeed be associated with low levels of synaptic coupling (Pumain and Heinemann 1985; Feng and Durand 2005, 2006). In these cases the authors report that the seizures occur in a low $\mathrm{Ca}^{++}$environment and that they are probably caused by a nonsynaptic process. The data provided by Feng and Durand, however, shows the presence of weak synaptic coupling at the seizure onset (for instance, in Fig. 2D in their 2004 paper). In adult mouse slices, low concentration $(\sim 5 \mu \mathrm{M})$ of CNQX (antagonist of the excitatory neurotransmitter AMPA) may cause repetitive or isolated bursting (van Drongelen et al., 2005). In slice experiments of mouse and rat neocortex, repetitive seizurelike activity has been observed upon application of $\mathrm{GABA}_{\mathrm{A}}$ antagonist bicuculline (van Drongelen et al. 2003; Khazipov et al. 2004). These repetitive oscillations were observed only in animals below 13-14 days of age in which the GABA causes depolarization (excitation) (Owens et al. 1996). In older rodents, bicuculline elicits bursts in cortical circuits but not in the typical seizurelike fashion; these bursts are perhaps more similar to the interictal spike. Activity patterns oscillating back and forth between cortical layers have also been observed experimentally in penicillin-evoked seizures in rabbits (Pockberger et al. 1984), and Pumain and Heinemann (1985) show that $\mathrm{Ca}^{++}$is low in the superficial and deep neocortical layers when evoking activity. Although the experimental evidence is circumstantial and evidence of the contrary exists, the data suggests that a number of seizure types may be associated with oscillations between cortical layers or reduced excitatory coupling.

\section{Acknowledgments}

We thank Drs. M. H. Kohrman, J.-M. Ramirez, and V. L. Towle for discussion and useful comments. Part of this work was supported by the U.S. Department of Energy under Contract W-31-109-Eng-38. We thank the Falk Foundation and the Linn Family for support. 


\section{References}

Amitai Y, Gibson JR, Beierlein M, Patrick SL, Ho AM, Connors BW, Golomb D. The spatial dimensions of electrically coupled networks of interneurons in the neocortex. J Neurosci 2002;22:4142-52.

Barela AJ, Waddy SP, Lickfett JG, Hunter J, Anido A, Helmers SL, Goldin AL, Escayg A. An epilepsy mutation in the sodium channel SCN1A that decreases channel excitability. J Neurosci. 2006;26:2714-23.

Bower JM, Beeman D. The book of GENESIS. New York: Springer-Verlag, 1998.

Destexhe A, Pare D. Impact of network activity on the integrative properties of neocortical pyramidal neurons in vivo. $J$ Neurophysiol 1999;81:1531-47.

DeFelipe J, Alonso-Nanclares L, Arellano JI. Microstructure of the neocortex: comparative aspects. J Neurocytol 2002;31:299-316.

Delgado-Escueta AV, Wilson WA, Olsen RW, Porter RJ. New waves of research in the epilepsies: crossing into the third millennium. In: Delgado-Escueta AV, Wilson WA, Olsen RW, Porter RJ, eds. Jasper's Basic Mechanisms of Epilepsies. Philadelphia: Lippincot, Williams \& Wilkins 1999;3-58. (Advances in neurology; vol 79.)

Feldmeyer D, Sakmann B. Synaptic efficacy and reliability of excitatory connections between principle neurones of the input (layer 4) and output (layer 5) of the neocortex. $J$ Physiol 2000;525:31-9. 
Feng Z, Durand DM. Propagation of low calcium non-synaptic induced epileptiform activity to the contralateral hippocampus in vivo. Brain Res 2005;1055:25-35.

Feng Z, Durand DM. Effects of potassium concentration on firing patterns of low-calcium epileptiform activity in anesthetized rat hippocampus: inducing of persistent spike activity. Epilepsia 2006;47:727-36.

Fleidervish IA, Friedman A, Gutnick MJ. Slow inactivation of $\mathrm{Na}^{+}$current and slow cumulative spike adaptation in mouse and guinea-pig neocortical neurones in slices. $J$ Physiol 1996;493:8397.

Golomb D. Models of neuronal transient synchrony during propagation of activity through neocortical circuitry. J Neurophysiol 1998;79:1-12.

Golomb D, Amitai Y. Propagating neuronal discharges in neocortical slices: computational and experimental study. J Neurophysiol 1997;78:1199-211.

Hellwig B. A quantitative analysis of the local connectivity between pyramidal neurons in layer 2/3 of the rat visual cortex. Biol Cybern 2000;82:111-21.

Hodgkin AL, Huxley AF. A quantitative description of membrane current and its application to conduction and excitation in nerve. J Physiol 1952;117:500-44.

Kepecs A, Wang XJ, Lisman J. Bursting neurons signal input slope. J Neurosci 2002;22:905362. 
Khazipov R, Khalilov I, Tyzio R, Morozova E, Ben-Ari Y, Holmes GL. Developmental changes in GABAergic actions and seizure susceptibility in the rat hippocampus. Eur $J$ Neurosci 2004;19:590-600.

Klopp JC, Johnston P, Nenov VI, Goddard N, Hood G and Halgren E. (1998) Large scale simulations of hippocampal-neocortical interactions in a parallel version of GENESIS. In: Bower JM. ed. Computational Neuroscience. New York: Plenum, 1998;59-64.

Krimer LS, Goldman-Rakic PS. Prefrontal microcircuits: Membrane properties and excitatory input of local, medium and wide arbor interneurons. J Neurosci 2001;21:3788-96.

Kwan P, Brodie MJ. Early identification of refractory epilepsy. N Engl J Med 2000;342:314-9.

Lee HC, Hereld M, Stevens R, Van Drongelen W. Epileptiform activity patterns in coupled neural networks. Invited paper at Joint Meeting of 5th International Conference on Bioelectromagnetism and 5th International Symposium on Noninvasive Functional Source Imaging within the Human Brain and Heart (BEM\&NFSI), Minneapolis, Minnesota, May 2005.

Lytton WW, Contreras D, Desthexe A and Steriade M. Dynamic interactions determine partial thalamic quiescence in a computer network model of spike-and-wave seizures. $J$ Neurophysiol 1997;77: 1679-96. 
Lytton WW, Sejnowski TJ. Simulations of cortical pyramidal neurons synchronized by inhibitory interneurons. J Neurophysiol 1991;66:1059-79.

Migliore M, Cannia C, Lytton WW, Markram H, Hines ML. Parallel network simulations with NEURON. J Comput Neurosci 2006 (in press).

Mountcastle VB. The columnar organization of the neocortex. Brain 1997;120:701-22.

Netoff TI, Schiff SJ. Decreased neural synchronization during experimental seizures. $J$ Neurosci 2002;22:7297-307.

Nieuwenhuys R. The neocortex. An overview of its evolutionary development, structural organization and synaptology. Anat Embryol 1994;190:307-37.

Nunez PL. Electric fields of the brain: the neurophysics of EEG. Oxford: Oxford University Press, 1981.

Owens DF, Boyce LH, Davis MBE, Kriegstein AR. Excitatory GABA responses in embryonic and neonatal cortical slices demonstrated by gramicidin perforated-patch recordings and calcium imaging. J Neurosci 1996;16,6414-23.

Pockberger H, Rappelsberger P, Petsche H. Penicillin-induced epileptic phenomena in the rabbit's neocortex II. Laminar specific generation of interictal spikes after the application of penicillin to different cortical depths. Brain Res 1984;309:261-9. 
Pumain R, Heinemann U. Stimulus- and amino acid-induced calcium and potassium changes in rat neocortex. J Neurophysiol 1985;53:1-16.

Pumain R, Menini C, Heinemann U, Louvel J, Silva-Barrat C. Chemical synaptic transmission is not necessary for epileptic seizures to persist in the baboon Papio papio. Exp Neurol 1985;89: $250-258$.

Steriade M, Amzica F. Intracellular study of excitability in the seizure-prone neocortex in vivo. $J$ Neurophysiol 1999;82:3108-22.

Tao JX, Ray A, Hawes-Ebersole S, Ebersole JS. Intracranial EEG substrates of scalp EEG interictal spikes. Epilepsia 2005;46:669-76.

Traub RD, Contreras D, Cunningham MO, Murray H, LeBeau FE, Roopun A, Bibbig A, Wilent WB, Higley MJ, Whittington MA. Single-column thalamocortical network model exhibiting gamma oscillations, sleep spindles, and epileptogenic bursts. J Neurophysiol 2005;93:2194-232.

Traub RD, Jefferys JGR, Miles, R, Whittington, MA and Toth K. A branching dendritic model of a rodent CA3 pyramidal neurone. J Physiol 1994;481: 79-95.

Traub RD, Llinas R. Hippocampal pyramidal cells: significance of dendritic ionic conductances for neuronal function and epileptogenesis. J Neurophysiol 1979;42, 476-96.

Traub RD, Miles R. Neural networks of the hippocampus. New York: Cambridge University Press, 1991. 
Van Drongelen W, Hecox K. Desynchronization of neural activity in a network model. Neurocomputing 2003;52-54: 425-30.

Van Drongelen W, Koch H, Marcuccilli C, Peña F, Ramirez J-M. Synchrony levels during evoked seizure-like bursts in mouse neocortical slices. J Neurophysiol 2003;90:1571-80.

Van Drongelen W, Koch H, Elsen F, Doren E, Lee H, Marcuccilli C, Hereld M, Stevens R, and Ramirez J-M - The role of persistent sodium current in bursting activity of mouse neocortical networks in vitro. $J$. Neurophysiol. in press.

Van Drongelen W, Lee HC, Hereld M, Jones D, Cohoon M, Elsen F, Papka ME, Stevens RL. Simulation of neocortical epileptiform activity using parallel computing. Neurocomputing 2004;58-60:1203-9.

Van Drongelen W, Lee HC, Koch, Hereld M, H, Elsen F, Chen Z, Stevens RL. Emergent epileptiform activity in neural networks with weak excitatory synapses. IEEE Trans. Neur. Sys. \& Rehab. 2005;13: 236-41.

Wang XJ. Fast burst firing and short-term synaptic plasticity: a model of neocortical chattering neurons. Neuroscience 1999;89:347-62.

Willis JB, Ge YC, Wheal HV. Simulation of epileptiform activity in the hippocampus using transputers. J Neurosci Methods 1993;47:205-13. 


\section{Figures \& Legends}

(A)

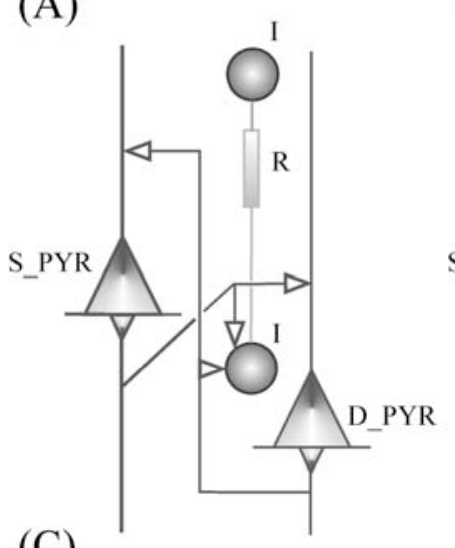

(B)

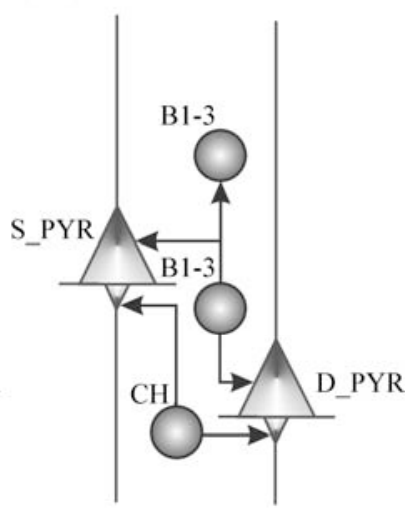

(C)

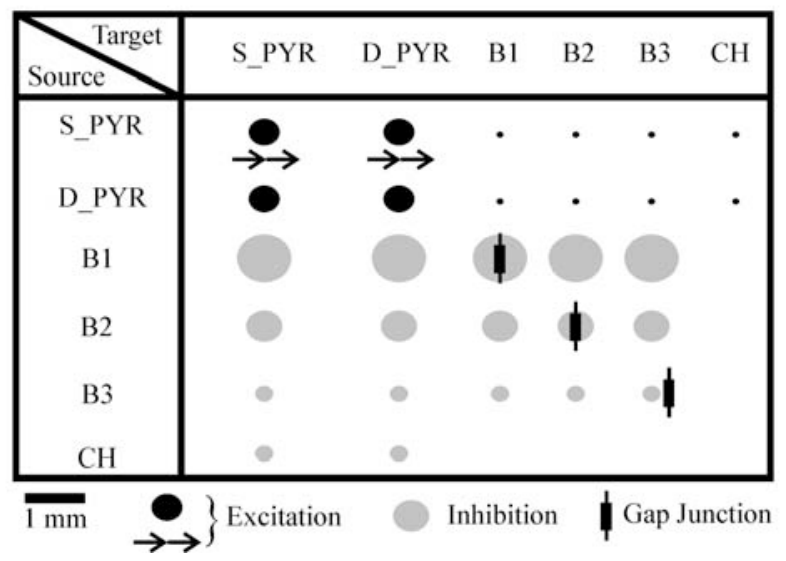

Fig. 1

Diagrams of the neocortical network model. The excitatory (A) and inhibitory (B) connectivity in the network of multicompartmental neuron models. Each neuron type is indicated: S_PYR and D_PYR - superficial and deep pyramidal neuron respectively; I - inhibitory cell, either a type of basket cell (B1-3) or a chandelier cell $(\mathrm{CH})$. The area of connectivity is depicted in the table in (C). The area for each type of synaptic connection is depicted by the size of the dot (see $1 \mathrm{~mm}$ scale); excitatory connections are dark dots, and inhibitory connections are light gray ones.

Within each area of connection, both the probability for connections and their strength decrease exponentially. Fast connections between the superficial pyramidal cells and pyramidal cells at a distance of $\sim 1 \mathrm{~mm}$ are indicated with arrows. Gap junctions between neighboring basket cells are represented by the resistor symbol. 

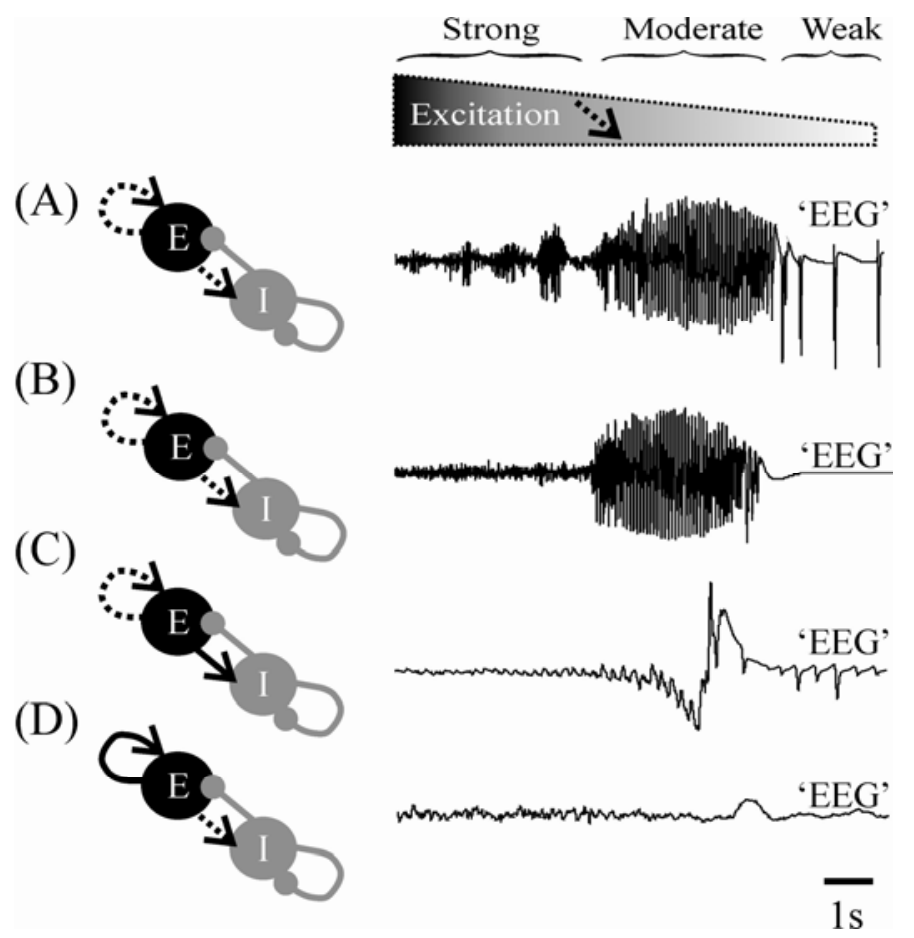

Fig. 2

Vertical oscillations in the model. A reduction of excitatory coupling strength generates oscillatory network activity that can be viewed in the compound 'EEG' signal (A). In both (A) and (B) all excitatory connections were reduced in strength. In trace (A) the network contained bursting neurons; in (B) this cell type was absent. In panel (C) the strength for the synaptic connections between the excitatory populations was selectively reduced; in trace (D) only the strength of the coupling between the excitatory and inhibitory populations was reduced.

Comparing traces (C), (D) with (A), one can see that both connections are critically associated with the network bursts and oscillations observed in trace (A). In both cases a selective reduction of part of the excitatory system abolishes the oscillatory activity - although, upon weakening the excitatory $\rightarrow$ excitatory connections, some oscillatory activity is retained (C). 


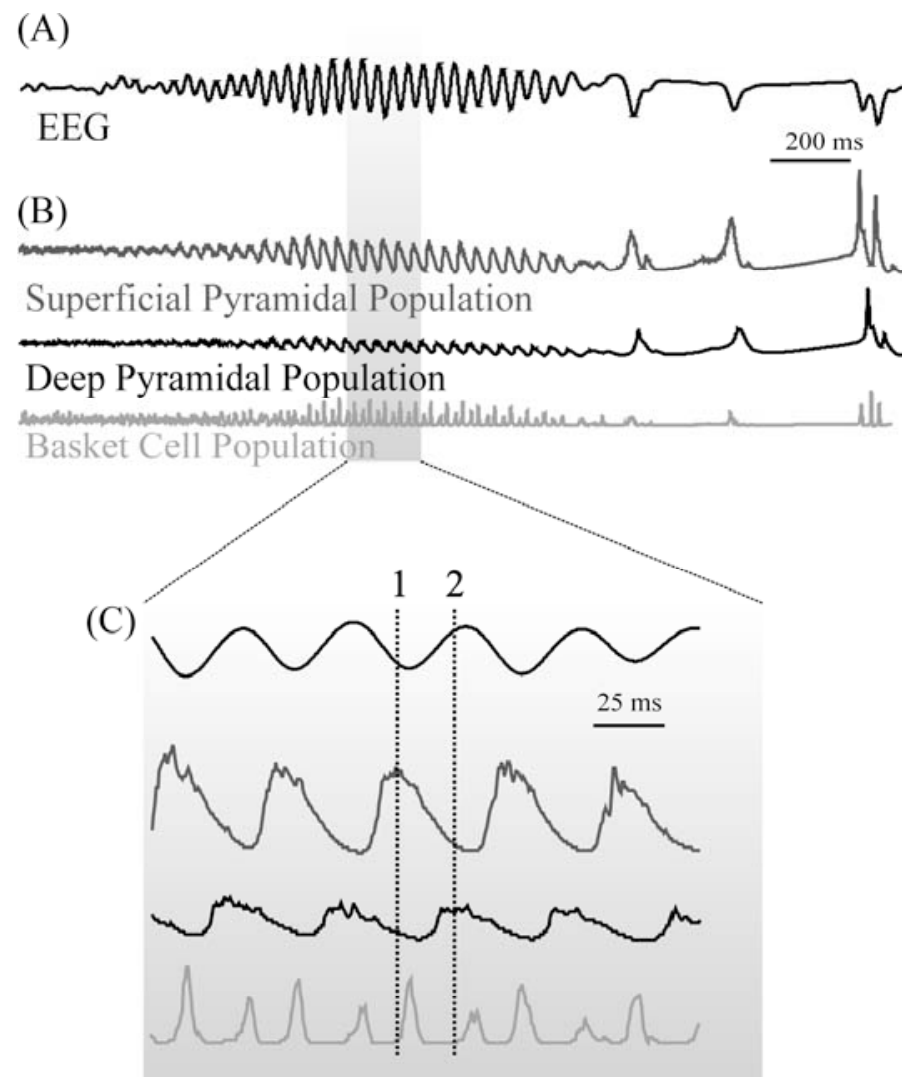

Fig. 3

Activity in the network during a reduction of excitatory coupling strength, represented by the compound electrical activity ('EEG'), is shown in (A). In panel (B), the associated compound activity for different neuronal populations is shown: the superficial and deep pyramidal cells plus the type 1 basket cells. A detail of this panel (gray area in A and B) is depicted in (C). It can be seen that the superficial and deep pyramidal layers almost alternate: when the superficial pyramidal cell population is maximally active, the deep pyramidal population's activity is decreasing (vertical dotted line 1), and when the deep pyramidal population's activity is high, the superficial pyramidal neuron's signal is low (vertical dotted line 2). Further, it can be seen that the inhibitory population is activated after both peaks in the pyramidal cell populations; the peak following the superficial cell activation is larger in amplitude compared to the one that follows the activation of the deep pyramidal cell layer. 


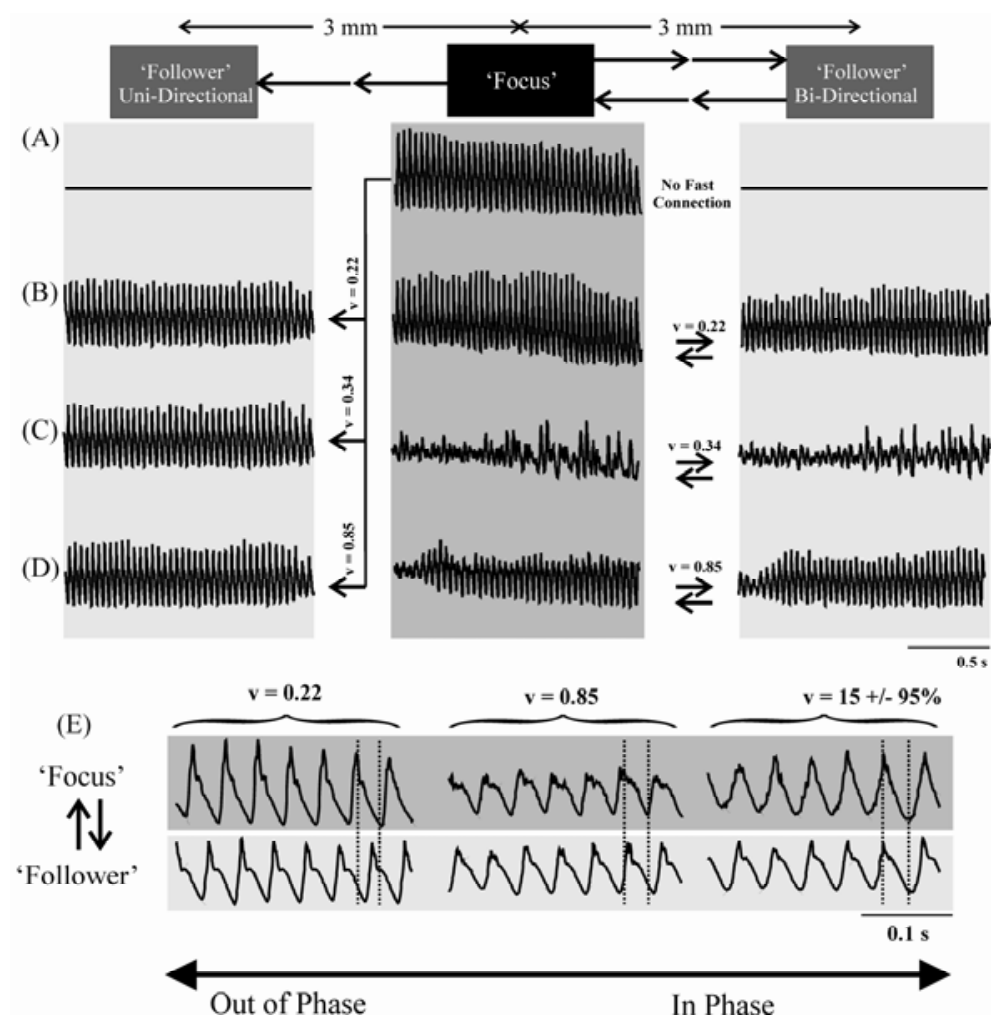

Fig. 4

Horizontal oscillations in a model of coupled patches of cortex. All traces in this figure are compound activity traces of the superficial pyramidal (S_PYR) cell populations. A focal area in which oscillations are set up by selecting the appropriate strength of the synaptic coupling (as shown in Figs. 2A and 3A) is connected to an area at a distance of $3 \mathrm{~mm}$. Because of the interdistance, the two areas are connected only by means of the faster intracortical fibers (Fig. 1C). When these fibers are absent, the follower patch remains inactive (A). For unidirectional coupling (left panels (A)-(D)) entrainment of the follower occurs independent of conduction velocity. For a bidirectional link (right panels (A)-(D)), the follower patch can become entrained by the focal one if the conduction speed is right. We show that this occurs at $\mathrm{v}=0.22 \mathrm{~m} / \mathrm{s}(\mathrm{B})$ and at $\mathrm{v}=0.85 \mathrm{~m} / \mathrm{s}(\mathrm{D})$. The oscillations in both the focal and follower patches are abolished at an intermediate speed of $0.34 \mathrm{~m} / \mathrm{s}(\mathrm{C})$. Details of the signals in the two bidirectionally coupled patches are shown in (E); here it can be seen that activity levels are out of phase at a speed of 0.22 $\mathrm{m} / \mathrm{s}$ and become more in phase at higher conduction velocities. 

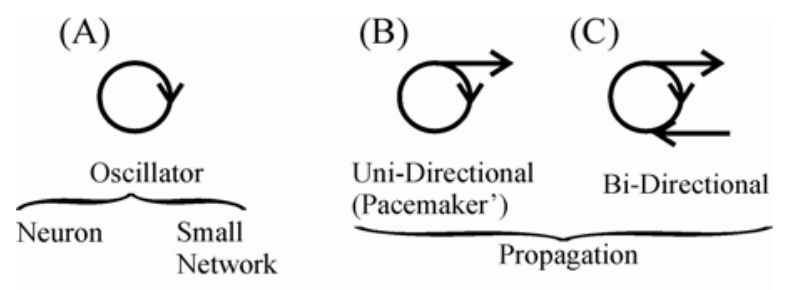

(D)

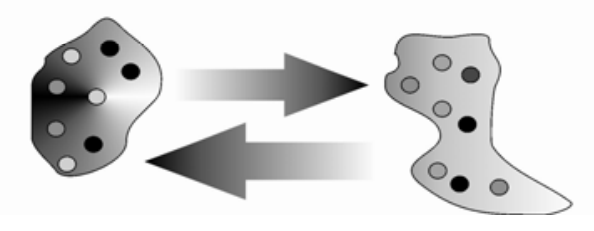

Fig. 5

Generalized diagrams representing sustained and propagating seizurelike activity observed in the model. The basis of the activity pattern is an oscillation that can be generated by a single neuron or neurons or by a small network. This activity pattern can propagate to a larger area in two ways: unidirectional (pacemaker) propagation, where the oscillation spreads through the tissue represented in (B), or a bi-irectional scenario in which the oscillation emits and receives a signal. In the case of a functional bidirectional connectivity (D), the frequency of the oscillatory activity can be somewhat flexible because (1) different neurons may be in a slightly different state of depolarization resulting in a range of latencies between activation and generation of an action potential, and (2) the fibers connecting the areas (represented by the arrows) may allow different conduction velocities between the populations. 


\begin{abstract}
The submitted manuscript has been created in part by the University of Chicago as Operator of Argonne National Laboratory ("Argonne") under Contract No. W31-109-ENG-38 with the U.S. Department of Energy. The U.S. Government retains for itself, and others acting on its behalf, a paid-up, nonexclusive, irrevocable worldwide license in said article to reproduce, prepare derivative works, distribute copies to the public, and perform publicly and display publicly, by or on behalf of the Government.
\end{abstract}

